\title{
АППРОКСИМАЦИЯ ОБЛИКА КРИСТАЛЛА ТРЁХОСНЫМ ЭЛЛИПСОИДОМ
}

\section{Степенщиков Д.Г.}

Геологический институт КНЦ РАН, Anamumbl, stepen@geoksc.apatity.ru

Рост кристалла в анизотропных условиях (неравномерный приток вещества, направленное воздействие, гравитация, ориентировка кристалла и т.п.), в общем случае, сопровождается неодинаковым развитием его граней. Получаемая при этом форма кристалла называется реальной [3] или искажённой (вынужденной, видимой, поверхностной) [5] и представляет интерес для генетической минералогии как индикатор симметрии кристаллообразующей среды. Фиксация такой формы может выполняться с разной степенью детализации несколькими взаимосвязанными способами [4], из которых самый распространённый - зарисовка или фотографирование образца. Этот способ, безусловно, нагляден, но «однобок» и порой излишне детализирован. В данной работе будет рассмотрен более упрощённый способ представления искажённой формы кристалла через его облик или габитус.
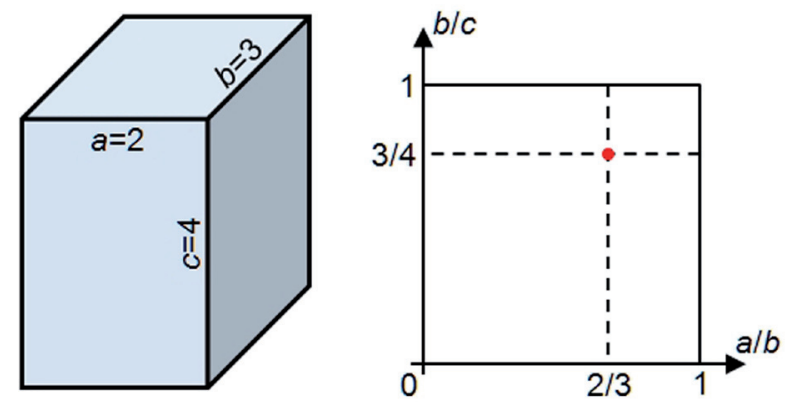

Рис. 1. Кристалл ромбической сингонии с измеренными параметрами и соответствующая его облику точка (красное) в единичном квадрате.

Согласно определению, облик кристалла оценивается по его линейным размерам $a, b$ и $c$, измеренным вдоль трёх направлений [1]. В этом есть определённый плюс, так как вся информация об облике заключена всего в трёх числах. Если рассматривать отношения этих чисел, то при условии, что $a \leq b \leq c$, облик можно представить наглядно в виде точки с координатами $(a / b, b / c)$ внутри единичного квадрата, (рис. 1). Но есть и сложности. Во-первых, выбор направлений, вдоль которых измеряются числа $a, b$ и $c$ неоднозначен. Они могут быть как сонаправлены, так и нет с элементами симметрии кристалла, быть взаимно ортогональными или нет (рис. 2 а, б). Во-вторых, сами величины могут быть измерены разными способами (рис. 2 б, в) или иметь определённую упорядоченность (например, может особо выделяться параметр, соответствующий главной оси в сингониях средней категории). В-третьих, при «неудачно» выбранных направлениях и способе измерения, одним и тем же измеренным величинам могут соответствовать разные облики (рис. 3).

Эти недостатки связаны с нестрогим определением облика (в [2] об этом сказано прямо), в котором нечётко указаны «три направления» и способ измерения «вытянутости» или «уплощённости». Точное определение облика должно корректно работать для всех случаев, то есть быть при-
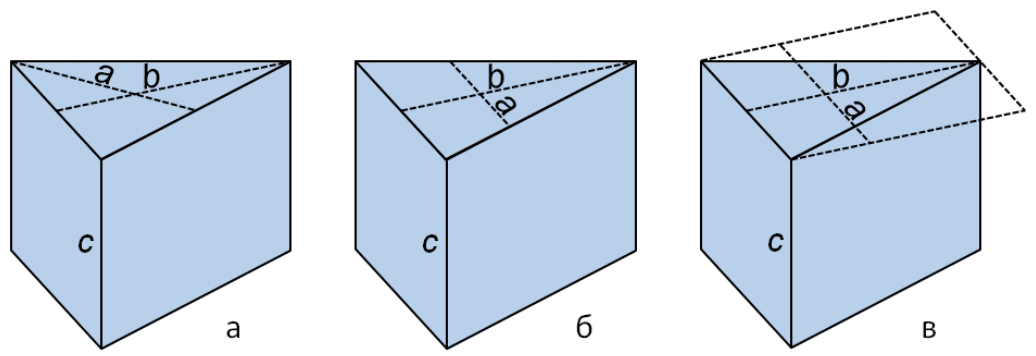

Рис. 2. Кристалл тригональной сингонии с различно измеренными величинами $a$ и $b$ : a - вдоль неперпендикулярных осей 2-го порядка, б - вдоль взаимно перпендикулярных направлений, между точками пересечения направлений с поверхностью кристалла, в - вдоль взаимно перпендикулярных направлений, между наиболее удаленными вдоль направления точками кристалла. 

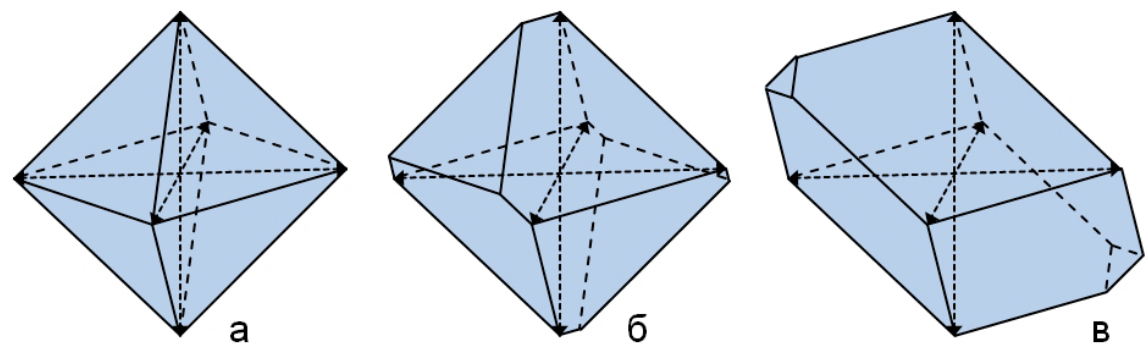

Рис. 3. а - идеальный октаэдрический кристалл, б, в - его искажённые формы. Все формы имеют одинаковые величины $a, b$ и $c$ измеренные вдоль осей 4-го порядка.

менимым к кристаллам любой сингонии и любой степени искажения, а также быть более или менее адекватным интуитивной оценке наблюдаемых форм. Так, для всех неискажённых простых форм кубической сингонии эта оценка должна констатировать строгую изометричность - равенство всех трёх параметров, а любое искажение этих форм должно тут же отводить оценку от строгой изометричности, чего нет, например, на рис. 3.

В данной работе предлагается заменить искажённую форму кристалла более простым геометрическим телом, а именно трёхосным эллипсоидом. Такой эллипсоид задаётся тремя параметрами - длинами своих взаимно перпендикулярных полуосей. Положение центра эллипсоида, длины его полуосей и их ориентировка выбираются таким образом, чтобы обеспечить «наибольшее приближение» формы эллипса к форме рассматриваемого искажённого кристалла. В качестве критерия, максимизирующего такое приближение (или, что то же самое, минимизирующего расхождение) предлагается использовать минимум симметрической разности объёмов эллипсоида и искажённой формы кристалла, т.е. сумму объёмов их непересекающихся частей (рис. 4). В идеале, если кристалл сам имеет эллипсоидную форму (например, кварцевая галька), то такая симметрическая разность равняется нулю.

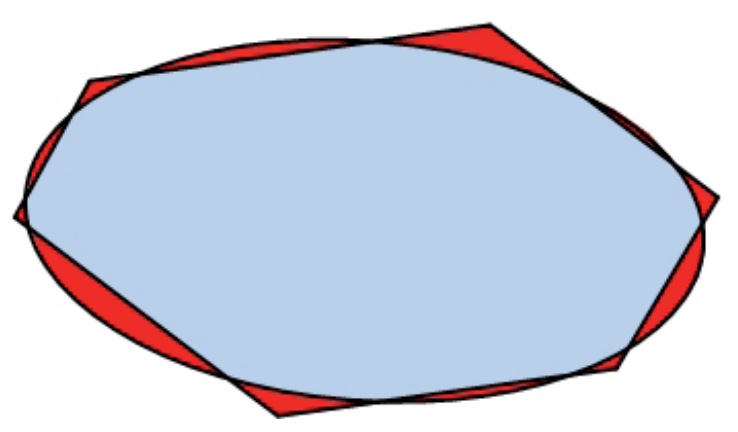

Рис. 4. Двухмерная проекция искажённого кристалла и совмещённого с ним эллипсоида. Симметрическая разность показана красным.

Данный подход отчасти подкрепляется его применением к искажённым кристаллам кубического габитуса, форма которых представляет собой, в общем случае, прямоугольный параллелепипед, и может быть описана его тремя параметрами - длиной, шириной и высотой. Предположим, что эллипсоид, аппроксимирующий искажённый кристалл, имеет с ним общий центр и оси, сонаправленные с осями 4-го порядка. Тогда в силу симметрии, достаточно рассмотреть симметрическую разность $V$ объёмов искажённой формы кристалла и эллипсоида только в одном октанте (например, положительном). Обозначим полудлину, полуширину и полувысоту параллелепипеда как $a, b$ и $c$, а соответствующие полуоси эллипсоида $-p, q$ и $r$ (рис. 5 ). Тогда верно равенство:

$$
V=a b c+2\left(V_{1}+V_{2}+V_{3}\right)-\frac{\pi}{6} p q r,
$$

где $V_{1}, V_{2}$ и $V_{3}$ - объёмы четвертей эллипсоидных сегментов вдоль осей $x, y$ и $z$ (рис. 5). 


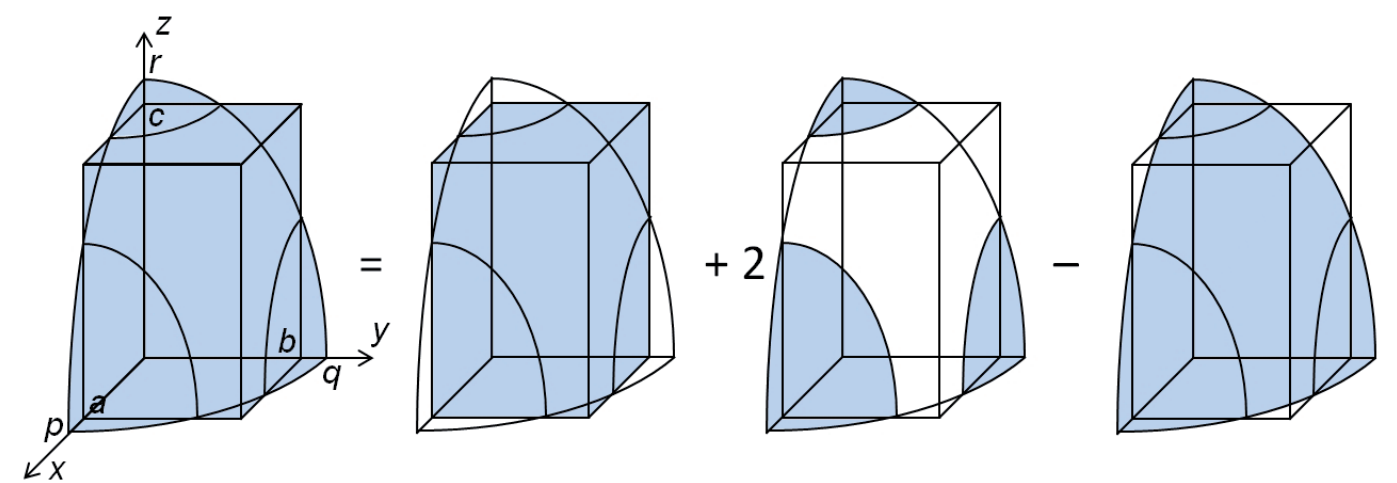

Рис. 5. Схема получения объёма симметрической разности параллелепипеда и аппроксимирующего его эллипсоида.

Найдём объём $V_{1}$ через интеграл:

$$
V_{1}=\frac{1}{4} \int_{a}^{p} S_{9} d x=\frac{1}{4} \int_{a}^{p} \pi q \sqrt{1-\frac{x^{2}}{p^{2}}} r \sqrt{1-\frac{x^{2}}{p^{2}}} d x=\frac{\pi q r}{4} \int_{a}^{p}\left(1-\frac{x^{2}}{p^{2}}\right) d x=\frac{\pi q r}{12}\left(2 p-3 a+\frac{a^{3}}{p^{2}}\right)
$$

Аналогично для $V_{2}$ и $V_{3}$ :

$$
V_{2}=\frac{\pi p r}{12}\left(2 q-3 b+\frac{b^{3}}{q^{2}}\right), \quad V_{3}=\frac{\pi p q}{12}\left(2 r-3 c+\frac{c^{3}}{r^{2}}\right)
$$

Подставляя полученные выражения в исходную формулу и упрощая выражение, получим:

$$
V=a b c+\frac{5 \pi}{6} p q r-\frac{\pi}{6}\left(3 a q r-\frac{q r a^{3}}{p^{2}}+3 b p r-\frac{p r b^{3}}{q^{2}}+3 c p q-\frac{p q c^{3}}{r^{2}}\right)
$$

Выясним, при каких $p, q$ и $r$ объём $V$ принимает минимальное значение. Найдём стационарные точки функции $V(p, q, r)$ :

$$
\left\{\begin{array} { l } 
{ \frac { \partial V } { \partial p } = \frac { \pi } { 6 } ( 5 q r - \frac { 2 q r a ^ { 3 } } { p ^ { 3 } } - 3 b r + \frac { r b ^ { 3 } } { q ^ { 2 } } - 3 c q + \frac { q c ^ { 3 } } { r ^ { 2 } } ) = 0 } \\
{ \frac { \partial V } { \partial q } = \frac { \pi } { 6 } ( 5 p r - 3 a r + \frac { r a ^ { 3 } } { p ^ { 2 } } - \frac { 2 p r b ^ { 3 } } { q ^ { 3 } } - 3 c p + \frac { p c ^ { 3 } } { r ^ { 2 } } ) = 0 } \\
{ \frac { \partial V } { \partial r } = \frac { \pi } { 6 } ( 5 p q - 3 a q + \frac { q a ^ { 3 } } { p ^ { 2 } } - 3 b p + \frac { p b ^ { 3 } } { q ^ { 2 } } - \frac { 2 p q c ^ { 3 } } { r ^ { 3 } } ) = 0 }
\end{array} \quad \Rightarrow \left\{\begin{array}{l}
5 q r-\frac{2 q r a^{3}}{p^{3}}-3 b r+\frac{r b^{3}}{q^{2}}-3 c q+\frac{q c^{3}}{r^{2}}=0 \\
5 p r-3 a r+\frac{r a^{3}}{p^{2}}-\frac{2 p r b^{3}}{q^{3}}-3 c p+\frac{p c^{3}}{r^{2}}=0 \\
5 p q-3 a q+\frac{q a^{3}}{p^{2}}-3 b p+\frac{p b^{3}}{q^{2}}-\frac{2 p q c^{3}}{r^{3}}=0
\end{array}\right.\right.
$$

Сделаем замену: $\frac{a}{p}=m, \frac{b}{q}=n, \frac{c}{r}=k$. Получим систему уравнений:

$$
\left\{\begin{array}{l}
\operatorname{qr}\left(5-2 m^{3}-3 n+n^{3}-3 k+k^{3}\right)=0 \\
\operatorname{pr}\left(5-3 m+m^{3}-2 n^{3}-3 k+k^{3}\right)=0 \\
\operatorname{pq}\left(5-3 m+m^{3}-3 n+n^{3}-2 k^{3}\right)=0
\end{array}\right.
$$

Учитывая, что $p, q$ и $r$ не могут быть нулевыми, имеем: 


$$
\left\{\begin{array}{l}
5-2 m^{3}-3 n+n^{3}-3 k+k^{3}=0 \\
5-3 m+m^{3}-2 n^{3}-3 k+k^{3}=0 \\
5-3 m+m^{3}-3 n+n^{3}-2 k^{3}=0
\end{array}\right.
$$

Складывая все три уравнения, получим равенство:

$$
m+n+k=\frac{5}{2}
$$

Вычитая из первого уравнения системы второе, из первого третье и из второго третье, получим систему:

$$
\left\{\begin{array}{l}
(m-n)\left(m^{2}+m n+n^{2}-1\right)=0 \\
(m-k)\left(m^{2}+m k+k^{2}-1\right)=0 \\
(n-k)\left(n^{2}+n k+k^{2}-1\right)=0
\end{array}\right.
$$

Данная система равносильна следующим случаям:

1) $(m-n)=(m-k)=0$. Отсюда следует, что $m=n=k=\frac{5}{6}$;

2) $(m-n)=0,\left(m^{2}+m k+k^{2}-1\right)=0$. Тогда $k=\frac{5}{2}-2 m$ и из второго равенства получим квадратное уравнение $4 m^{2}-10 m+7=0$, которое не имеет действительных корней;

3) $\left(m^{2}+m n+n^{2}-1\right)=\left(m^{2}+m k+k^{2}-1\right)=0$. Вычтем из первого уравнения второе и, преобразуя выражение, получим $(n-k)(m+n+k)=0$. Это равенство выполняется только при $n=k$, что автоматически сводится к первому или второму случаю.

Таким образом, мы имеем единственную точку экстремума $\left(\frac{6}{5} a, \frac{6}{5} b, \frac{6}{5} c\right)$ функции $V(p, q, r)$, при $m=n=k=\frac{5}{6}$. Она является точкой минимума, что легко проверить, подставляя в исходную формулу координаты этой точки, и любой другой, например точки $(a, b, c)$ - в последней значение функции $V(p, q, r)$ будет больше. Главный вывод заключается в том, что полученные значения полуосей аппроксимирующего эллипсоида относятся друг к другу так же, как и параметры аппроксимируемого параллелепипеда, а значит им, в частности, будет соответствовать одна и та же точка в единичном квадрате (рис. 1). Параметры эллипсоида в абсолютном выражении пропорционально увеличены в 1.2 раза.

Рассмотренный пример, основан на предположении об известных ориентировках осей эллипсоида и положении его центра. Строгое доказательство того, что подобная установка даёт минимум $V$, требует более громоздкого доказательства. Ситуация усугубляется при переходе к более сложным формам, для которых облики интуитивно понятны (например, все формы кубической сингонии должны аппроксимироваться сферами). В общем случае, требуется найти 9 неизвестных параметров (координаты центра эллипсоида, ориентировки осей и длины полуосей), из которых практически информативны только три последних.

Вычислительная сложность метода, как представляется автору, может быть преодолена созданием эффективных алгоритмов и компьютерных программ, позволяющих по уже разработанным способам представления форм кристаллов находить параметры аппроксимирующего их эллипса.

Работа выполнена в рамках темы НИР ГИ КНЦ РАН № 0231-2015-0001.

\section{Литература}

1. Бетехтин А.Г. Курс минералогии: учебное пособие / А.Г. Бетехтин; под науч. ред. Б.И. Пирогова и Б.Б. Шкурского. 2-е изд., испр. и доп. М.: КДУ. 2010. 736 с.

2. Булах А.Г. Общая минералогия: учебник для студ. высш. учеб. заведений / А.Г. Булах, В.Г. Кривовичев, А.А. Золотарёв. 4-е изд., перераб. и доп. М.: Изд. центр «Академия». 2008. 416 с.

3. Войтеховский Ю.Л., Степенщиков Д.Г. Реальные кристаллографические простые формы // ЗВМО. № 2. 2004. С. 112-120.

4. Степенщиков Д.Г. О внешней симметрии кристалла // Федоровская сессия 2010. Матер. конф. СПб. 2010. С. $152-154$.

5. Шафрановский И.И., Корень Р.В., Дубов П.Л. К методике изучения искажённых форм на кристаллах минералов // Зап. Всесоюзн. Мин. общ. 1971. Т. 100. Вып. 1. С. $42-48$. 